\title{
DYNAMICAL INTERACTION OF AN ELASTIC SYSTEM AND A VIBRO-IMPACT ABSORBER
}

\author{
YURI V. MIKHLIN AND S. N. RESHETNIKOVA
}

Received 15 December 2004; Revised 9 August 2005; Accepted 22 August 2005

The nonlinear two-degree-of-freedom system under consideration consists of the linear oscillator with a relatively big mass, which is an approximation of some continuous elastic system, and of the vibro-impact oscillator with a relatively small mass, which is an absorber of the linear system vibrations. Analysis of nonlinear normal vibration modes shows that a stable localized vibration mode, which provides the vibration regime appropriate for the elastic vibration absorption, exists in a large region of the system parameters. In this regime, amplitudes of vibrations of the linear system are small, simultaneously vibrations of the absorber are significant.

Copyright (c) 2006 Y. V. Mikhlin and S. N. Reshetnikova. This is an open access article distributed under the Creative Commons Attribution License, which permits unrestricted use, distribution, and reproduction in any medium, provided the original work is properly cited.

\section{Introduction}

Numerous scientific papers contain a description and analysis of different devices for the absorption of elastic vibrations due to the importance of these problems in engineering. It is known that in many cases the absorption can be effective by using linear absorbers with big masses, but this is impossible to realize in most concrete systems. So, an analysis of absorption by using nonlinear passive absorbers is interesting for both theory and engineering applications. Here some publications on the subject are selected. In particular, principal aspects of the nonlinear absorption theory are analyzed by Kolovski [11]. The linear and nonlinear absorber general theory is presented too in the handbook [5]. Haxton and Barr [7] analyzed the absorber in the form of a beam, which is attached to the linear mass-spring system. An existence of a transfer of energy from the periodic forcing of the mass-spring system into the beam was shown. The pendulum-type centrifugal absorber was analyzed in numerous papers. Shaw and Wiggins [22] analyzed such type of absorber to reduce torsion oscillations. Lee and Shaw [12] considered a quenching of torsion oscillations of the internal combustion engine by

Hindawi Publishing Corporation

Mathematical Problems in Engineering

Volume 2006, Article ID 37980, Pages 1-15

DOI 10.1155/MPE/2006/37980 
this type of absorber too. Haddow and Shaw [6] studied experimentally the rotating machinery with the centrifugal pendulum absorber. Different aspects of the use of pendulum absorbers were also considered in $[4,26]$. Natsiavas [19] offered to use the oscillator with a nonlinear spring to absorb forced oscillations of the Duffing system. The mass-spring nonlinear system to reduce vibrations of some self-excited system was analyzed in [20]. Impact systems which can be used to absorb oscillations are analyzed by Karyeaclis and Caughey $[9,10]$. Aoki and Watanabe [1] offered the impact absorber, which contains small mass hitting on the stop. In [24] the process of redistribution of energy was considered in a system of connected linear and nonlinear oscillators. The energy transfer to nonlinear normal mode is caused by subharmonic resonance which is possible because of the nonlinear oscillator existence. In other papers by Vakakis et al. [14, 15], theoretical investigation and some experimental verification on the use of nonlinear localization for reducing the transmitted vibrations in structures subjected to transient base motions have been presented. In particular, the experimental assembly, containing the main linear subsystem and the nonlinear absorber, is described in [15]. It was shown that the energy transfer to the nonlinear absorber exists. A semi-infinite linear chain with an essentially nonlinear absorber is considered in [23].

In this paper, the single-DOF vibro-impact oscillator is examined to quench oscillations of some elastic system. In this case, a part of the elastic oscillation energy is transferred to the oscillator. The elastic structure is approximated by the single-DOF massspring model to study the principal capacity of the oscillation absorption. The mass and stiffness of the absorber are smaller than the corresponding parameters of the main linear system. Such choice of the parameter is determined by the real engineering design conditions. Oscillations of the two-DOF system are studied by methods of the nonlinear normal vibration mode (NNM) theory $[13,16,25]$. It is possible to select in the two-DOF nonlinear system under consideration the nonlocalized normal mode when the vibration amplitudes of the main linear subsystem and essentially nonlinear absorber are comparable, and the localized normal mode. The localized NNM, when the main linear system and absorber have small and large vibration amplitudes, respectively, is appropriate for the absorption. Note that the NNM approach was used earlier in some problems of the linear vibration absorption in systems containing the absorber with a cubic nonlinearity and the snap-through truss as an absorber $[2,3,17]$.

Free vibrations of the system under consideration are analyzed in Section 2 by using the nonlinear normal vibration mode theory and the nonsmooth transformation approach by Pilipchuk. Analysis of stability of the localized and nonlocalized NNMs is presented in Section 3. The authors use the algebraization by Ince which can be successfully used in conservative systems to solve the stability problem. The regions in the system parameter space, where the nonlocalized mode is unstable and the localized mode, appropriate to the absorption, is stable are selected.

\section{Nonlinear normal modes in a system containing the vibro-impact absorber}

One considers a possibility to absorb vibrations of linear elastic structure. The singleDOF vibro-impact oscillator is examined as the absorber. To simplify the investigation, 


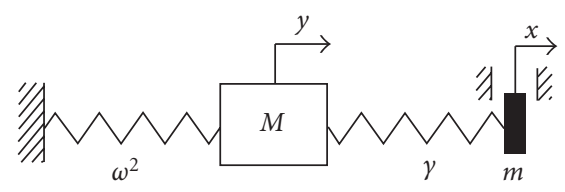

Figure 2.1. Two-DOF nonlinear system under consideration.

we replace the elastic system by a single-DOF linear oscillator. The corresponding transformation can be realized, for example, by using the standard Bubnov-Galerkin procedure. As a result, the following two-DOF nonlinear system (Figure 2.1) is investigated:

$$
\begin{gathered}
\varepsilon m \ddot{x}+\gamma(x-y)+P(x)=0, \\
M \ddot{y}+\omega^{2} y+\gamma(y-x)=0 .
\end{gathered}
$$

Here $x$ and $y$ are displacements of the absorber and main elastic systems, respectively; $\omega^{2}$ and $\gamma$ are stiffness coefficients of the springs. By assumption of Section 1, the mass of the absorber is significantly smaller than that of the elastic system. Therefore, the formal small parameter $\varepsilon$ is introduced. The function $P(x)$ describes the impact interaction of the absorber with the catch. The restoring force exerted by the catch is assumed in the form of the power function with the sufficiently big power. Considering only the impact interaction, we can present the absorber dynamics as a motion in the potential well with the potential in the following form: $\prod=x^{n+1} /(n+1)$, where the well becomes rectangular if $n \rightarrow \infty$. The authors consider here the elastic impact, so the restitution coefficient is equal to one.

In this system, both nonlocalized and localized vibration modes are possible. To analyze the vibration modes, methods of the NNM theory are used [13, 16, 25]. Nonlinear normal modes are a generalization of the normal (principal) vibrations of linear systems. In the mode, a finite-dimensional system behaves like a conservative one having a single degree of freedom, and all position coordinates can be analytically represented by any one of them.

One writes the system (2.1) energy integral of the form

$$
\mathrm{T}+\prod \equiv \varepsilon m \frac{\dot{x}^{2}}{2}+M \frac{\dot{y}^{2}}{2}+\omega^{2} \frac{y^{2}}{2}+\gamma \frac{(x-y)^{2}}{2}+\frac{x^{n+1}}{n+1}=h
$$

where $\mathrm{T}$ and $\Pi$ are kinetic and potential energies, respectively; $h$ is the system total energy.

Trajectories of the NNMs in the system (2.1) configuration space are sought in the form $y(x)$. The next relations to eliminate $t$ from (2.1) are used:

$$
\frac{d(\circ)}{d t}=\dot{x} \frac{d(\circ)}{d x}, \quad \frac{d^{2}(\circ)}{d t^{2}}=\dot{x}^{2} \frac{d^{2}(\circ)}{d x^{2}}+\ddot{x} \frac{d(\circ)}{d x} .
$$


Using these relations and the system energy integral (2.2), one derives the following equation to obtain the trajectories:

$$
\begin{aligned}
& M\left[2 y^{\prime \prime} \frac{h-\left(\omega^{2}\left(y^{2} / 2\right)+\gamma\left((x-y)^{2} / 2\right)+x^{n+1} /(n+1)\right)}{\varepsilon m+M\left(y^{\prime}\right)^{2}}-\frac{y^{\prime}}{\varepsilon m}\left(\gamma(x-y)+x^{n}\right)\right] \\
& \quad+\omega^{2} y+\gamma(y-x)=0 .
\end{aligned}
$$

Here prime means a derivation by $x$.

Equation (2.4) has singularities at the maximum equipotential surface $\Pi=h$ where, $x=X_{0}, y=y\left(X_{0}\right)$, and all velocities are equal to zero. The NNM trajectory can be analytically continued up to the maximum equipotential surface by satisfying some additional boundary condition,

$$
-M y^{\prime}\left(\gamma(x-y)+x^{n}\right)+\varepsilon m\left[\omega^{2} y+\gamma(y-x)\right]=0 \quad \text { if } x=X_{0},
$$

which is a condition of orthogonality of the NNM trajectory to the maximum equipotential surface $[13,16,25]$.

The zero approximation with respect to $\varepsilon(\varepsilon=0)$ gives us the following:

$$
y_{0}=x+\frac{x^{n}}{\gamma}
$$

This is the nonlocalized vibration mode. In this mode, the vibration energy is distributed both in the linear oscillator and in the essentially nonlinear absorber, that is, the vibration amplitudes of the subsystems are comparable. The corresponding limiting system, which can be obtained from (2.1), is the following:

$$
\gamma(x-y)+x^{n}=0, \quad M \ddot{y}+\omega^{2} y+\gamma(y-x)=0 .
$$

By using the classical procedure of the small parameter method, we can obtain the solution as the power series with respect to $\varepsilon$. Note that the power series method was proposed for a construction of the NNM curvilinear trajectories in $[13,16,25]$. The first approximation (by $\varepsilon$ ) equation and the corresponding boundary conditions are not presented here, but the equation can be easily obtained from the relations (2.4) and (2.5). The solution of the first approximation by $\varepsilon$ can be obtained in power series by $x$.

One selects now the localized vibration mode when amplitudes of vibrations of the main linear system are small; simultaneously vibrations of the absorber are significant. This regime can be analyzed if the next time transformation is imputed: $t=\sqrt{\varepsilon} \tau$. Then the system (2.1) can be written as

$$
m \ddot{x}+\gamma(x-y)+x^{n}=0, \quad \frac{M}{\varepsilon} \ddot{y}+\omega^{2} y+\gamma(y-x)=0 .
$$

The corresponding limiting system (for $\varepsilon \rightarrow 0$ ) has the form

$$
\begin{gathered}
m \ddot{x}+\gamma(x-y)+x^{n}=0, \\
M \ddot{y}=0 .
\end{gathered}
$$


One has from here $y_{0}=0$. In this case, the equation to obtain NNM trajectory $y(x)$ is the following:

$$
\begin{aligned}
& M\left[2 y^{\prime \prime} \frac{h-\left(x^{n+1} /(n+1)+\omega^{2}\left(y^{2} / 2\right)+\gamma\left((x-y)^{2} / 2\right)\right)}{m+(M / \varepsilon)\left(y^{\prime}\right)^{2}}+\frac{y^{\prime}}{m}\left(-x^{n}-\gamma(x-y)\right)\right] \\
& \quad+\varepsilon \omega^{2} y+\varepsilon \gamma(y-x)=0 .
\end{aligned}
$$

By using the first approximation equation with respect to $\varepsilon$ and the corresponding boundary conditions at the maximal equipotential surface, it is possible to obtain a solution of the form of the following power series by $x$.

One considers a new zero approximation (2.6) of the nonlocalized vibration mode. Substituting both the approximation of the function $y(x)$ and derivatives of the function by time, $\dot{y}=\left(1+(n / \gamma) x^{n-1}\right) \dot{x}$ and $\ddot{y}=\left(1+(n / \gamma) x^{n-1}\right) \ddot{x}+\left(n(n-1) x^{n-2} / \gamma\right) \dot{x}^{2}$, into the second equation of the system (2.1), one obtains the following equation to determine the solution $x(t)$ :

$$
M\left[\left(1+\frac{n}{\gamma} x^{n-1}\right) \ddot{x}+\frac{n(n-1) x^{n-2}}{\gamma} \dot{x}^{2}\right]+\omega^{2}\left(x+\frac{1}{\gamma} x^{n}\right)+x^{n}=0 .
$$

By expressing $\dot{x}^{2}$ from the system energy integral (2.2) and substituting it into (2.11), one has, as a resul $t$, the next equation:

$$
\begin{aligned}
M(1+ & \left.\frac{n}{\gamma} x^{n-1}\right) \ddot{x}+2 \frac{n(n-1)}{\gamma} \cdot \frac{h-\left[\left(\omega^{2} / 2\right)\left(x+(1 / \gamma) x^{n}\right)^{2}+x^{2 n} / 2 \gamma+x^{n+1} /(n+1)\right]}{\left(1+(n / \gamma) x^{n-1}\right)^{2}} x^{n-2} \\
& +\omega^{2}\left(x+\frac{1}{\gamma} x^{n}\right)+x^{n}=0 .
\end{aligned}
$$

According to the nonsmooth transformation theory developed by Pilipchuk $[21,25]$, one presents the periodic solution of the form

$$
x=A \tau+X(\tau), \quad \tau=\frac{2}{\pi} \arcsin \left(\sin \left(\frac{\pi}{2} \omega_{0} t\right)\right)=\tau\left(\omega_{0} t\right),
$$

where the function $X(\tau)$ has to be determined, and the parameters $A$ and $\omega_{0}$ will be connected later by the amplitude-frequency characteristic. One introduces now the new independent variable $\tau$ instead of the variable $t$ by using the formula (2.13). In this case, $\dot{x}=\omega_{0}\left(A+X^{\prime}\right) \dot{\tau}, \ddot{x}=\omega_{0}^{2}\left(A+X^{\prime}\right) \ddot{\tau}+\omega_{0}^{2} X^{\prime \prime}(\dot{\tau})^{2}=\omega_{0}^{2}\left(A+X^{\prime}\right) \ddot{\tau}+\omega_{0}^{2} X^{\prime \prime}$. Then one derives 




Figure 2.2. Amplitude-frequency relation, corresponding to the nonlocalized vibration mode.

the following equation instead of (2.12):

$$
\begin{aligned}
X^{\prime \prime}= & -2 \frac{n(n-1)}{M \gamma \omega_{0}^{2}} \\
& \cdot \frac{h-\left[\left(\omega^{2} / 2\right)\left(A \tau+X+(1 / \gamma)(A \tau+X)^{n}\right)^{2}+(A \tau+X)^{2 n} / 2 \gamma+(A \tau+X)^{n+1} /(n+1)\right]}{\left(1+(n / \gamma)(A \tau+X)^{n-1}\right)^{3}} \\
& \cdot(A \tau+X)^{n-2}-\frac{\omega^{2}\left(A \tau+X+(1 / \gamma)(A \tau+X)^{n}\right)+(A \tau+X)^{n}}{M \omega_{0}^{2}\left(1+(n / \gamma)(A \tau+X)^{n-1}\right)} .
\end{aligned}
$$

One supplements also the condition

$$
\left.X^{\prime}\right|_{\tau=1}+A=0
$$

which is a condition of elimination of singularity in (2.14). Here the new independent variable is limited, $|\tau| \leq 1$. The condition (2.15) permits to find out a solution of (2.14) without nonperiodic terms, by using the simple iterative procedures. One of them is described in [21]. By means of this procedure, the next amplitude-frequency relation was obtained: $M \omega_{0}^{2}=\left(\omega^{2} / 2\right)\left(1+(1 / \gamma) A^{n-1}\right)^{2}+A^{2(n-1)} / 2 \gamma+A^{n-1} /(n+1)$. This amplitudefrequency relation, corresponding to the nonlocalized vibration mode, is presented for different values of the index $n$ in Figure 2.2.

Similar calculations for the localized mode give us the relation $m \omega_{0}^{2}=\gamma / 2+A^{n-1} /$ $(n+1)$, which is presented in Figure 2.3.

\section{Stability of localized and nonlocalized nonlinear normal modes}

To investigate the stability of the system (2.1) solutions, a system of the variational equations can be written. Let $x=x_{0}+u, y=y_{0}+v$, where $u$ and $v$ are variations for the NNMs 


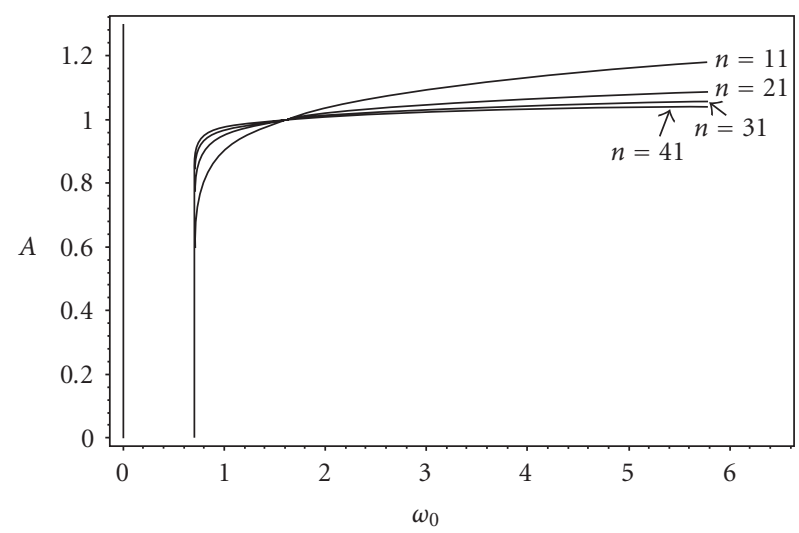

Figure 2.3. Amplitude-frequency relation, corresponding to the localized vibration mode.

of the system (2.1). Then we have

$$
\varepsilon m \ddot{u}+\gamma(u-v)+n x_{0}^{n-1} u=0, \quad M \ddot{v}+\omega^{2} v+\gamma(v-u)=0 .
$$

Note that we will investigate a stability of NNMs up to terms of the order $O(\varepsilon)$. In this case, eliminating the variable $u$ from the first equation (3.1), $u=v /\left(1+(n / \gamma) x_{0}^{n-1}\right)$, one obtains from the second equation (3.1) the following simplified variational equation:

$$
M \ddot{v}+v\left[\omega^{2}+\gamma\left(1-\frac{1}{1+(n / \gamma) x^{n-1}}\right)\right]=0 .
$$

First, one considers a stability of the nonlocalized vibration mode. A motion along this mode is determined by (2.11), which were obtained by using the approximate expression of the mode in the form (2.6). Note that a harmonic approximation of solution is impossible for the vibro-impact system under consideration.

The NNM stability analysis is based on the so-called algebraization by Ince. In this case, a new variable associated with the solution under consideration is chosen as an independent argument [8]. Then the variational equation is converted to the equation with singular points. This approach was used earlier to investigate a problem of the NNM stability $[13,18,25]$. Note that the Ince algebraization can be used to solve the stability problems only in conservative systems.

One introduces the following transformation of the independent variable: $t \rightarrow x$. Expressing the time derivatives in terms of the new independent variable $x$, namely, the relations (2.3), we can obtain the next equation instead of (3.2):

$$
M\left(v^{\prime \prime} \dot{x}^{2}+v^{\prime} \ddot{x}\right)+v\left[\omega^{2}+\gamma\left(1-\frac{1}{1+(n / \gamma) x^{n-1}}\right)\right]=0 .
$$


8 Elastic system and a vibro-impact absorber

Substituting the expressions, obtained previously for $\dot{x}^{2}$ and $\ddot{x}$, into (3.3), after some transformations, the following equation can be written as

$$
C_{0} v^{\prime \prime}-C_{1} v^{\prime}+C_{2} v=0
$$

where

$$
\begin{gathered}
C_{0}=2 \frac{h-\left[\left(\omega^{2} / 2\right)\left(x+(1 / \gamma) x^{n}\right)^{2}+x^{2 n} / 2 \gamma+x^{n+1} /(n+1)\right]}{\left(1+(n / \gamma) x^{n-1}\right)^{2}}, \\
C_{1}=2 \frac{n(n-1)}{\gamma} \cdot x^{n-2} \cdot \frac{h-\left[\left(\omega^{2} / 2\right)\left(x+(1 / \gamma) x^{n}\right)^{2}+x^{2 n} / 2 \gamma+x^{n+1} /(n+1)\right]}{\left(1+(n / \gamma) x^{n-1}\right)^{3}} \\
+\frac{\omega^{2}\left(x+(1 / \gamma) x^{n}\right)+x^{n}}{1+(n / \gamma) x^{n-1}}, \\
C_{2}=\frac{\omega^{2}\left(1+(n / \gamma) x^{n-1}\right)+n x^{n-1}}{1+(n / \gamma) x^{n-1}} .
\end{gathered}
$$

Singular points of (3.4) are situated on the maximal isoenergetic surface: $h-\left[\left(\omega^{2} / 2\right)(x+\right.$ $\left.\left.(1 / \gamma) x^{n}\right)^{2}+x^{2 n} / 2 \gamma+x^{n+1} /(n+1)\right]=0$. One denotes the points as $\pm X_{0}$. Indices of the equation singular points are equal to $\alpha_{1}=0$ and $\alpha_{2}=1 / 2$. It is demonstrated in [8] that solutions corresponding to boundaries of stability/instability regions of equations with singular points, in which indices are equal to 0 and $1 / 2$, are the following:

$$
\begin{gathered}
v_{1}=a_{0}+a_{1} x+a_{2} x^{2}+a_{3} x^{3}+a_{4} x^{4}+\cdots, \\
v_{2}=\sqrt{X_{0}^{2}-x^{2}} \cdot\left(b_{0}+b_{1} x+b_{2} x^{2}+b_{3} x^{3}+b_{4} x^{4}+\cdots\right) .
\end{gathered}
$$

Substituting the series $v_{1}$ into the variational equation (3.4) and matching respective powers of $x$, it is possible to obtain the next infinite recurrent system of linear algebraic equations to determine coefficients of the expansion (3.6):

$$
\begin{aligned}
& x^{0}: 4 h a_{2}+\omega^{2} a_{0}=0, \\
& x^{1}: 12 h a_{3}=0, \\
& x^{2}:-3 \omega^{2} a_{2}+24 h a_{4}=0, \\
& x^{3}: 40 h a_{5}-8 \omega^{2} a_{3}=0,
\end{aligned}
$$

It is clear that the system decomposes into two subsystems to determine coefficients with even and odd subscripts. 
Analogously, substituting the solution $v_{2}$ into (3.7) and matching respective powers of $x$, one has the following recurrent equations:

$$
\begin{aligned}
& x^{0}:-2 X_{0}^{2} h b_{0}+4 X_{0}^{4} h b_{2}=0, \\
& x^{1}: 12 X_{0}^{4} h b_{3}-6 X_{0}^{2} h b_{1}=0 \\
& x^{2}:-3 X_{0}^{4} \omega^{2} b_{2}-18 X_{0}^{2} h b_{2}+24 X_{0}^{4} h b_{2}=0, \\
& x^{3}: 40 X_{0}^{4} h b_{5}-38 X_{0}^{2} h b_{3}-8 X_{0}^{4} \omega^{2} b_{3}-2 X_{0}^{2} \omega^{2} b_{1}+4 h b_{1}=0,
\end{aligned}
$$

which also decompose into two subsystems to determine coefficients with even and odd subscripts.

As a result, four systems of the algebraic equations with respect to $a_{i}, b_{i}, i=\overline{1, \infty}$, are derived. These systems have nontrivial solutions if their determinants are equal to zero. These determinants were calculated up to sixth order. Thus four equations connecting the system parameters are derived. These equations give the instability region boundaries for the nonlocalized vibration mode. Three determinants have solutions only when the system parameters are equal to zero. The boundaries of instability for the last determinant, which correspond to $b_{i}, i=2 k, k \in N$, for the following parameters: $\varepsilon m=0.1, M=1$, $\omega^{2}=1$, are shown in Figure 3.1. The regions of instability are shaded in this figure.

Now the procedure of algebraization is used to analyze the stability of the localized vibration mode. It restricts oneself to the zero approximation of the mode: $y \cong 0$. The energy integral along this vibration mode has the form

$$
m \frac{\dot{x}^{2}}{2}+\gamma \frac{x^{2}}{2}+\frac{x^{n+1}}{n+1}=h
$$

Let us exclude from the first equation of the system (2.9) $\ddot{x}=-\left(\gamma x+x^{n}\right) / m$, and from the energy integral (3.10) $\dot{x}^{2}=2\left(h-\left(\gamma x^{2} / 2+x^{n+1} /(n+1)\right) / m\right)$. The results are substituted into the variational equation (2.14). One has the following equation:

$$
\begin{aligned}
\frac{2}{\mu}(h & \left.-\left[\frac{\gamma x^{2}}{2}+\frac{x^{n+1}}{n+1}\right]\right)\left(1+\frac{n x^{n-1}}{\gamma}\right) v^{\prime \prime}-\frac{1}{\mu}\left(\gamma x+x^{n-1}\right)\left(1+\frac{n x^{n-1}}{\gamma}\right) v^{\prime} \\
& +\left(\omega^{2}+n x^{n-1}\right) v=0,
\end{aligned}
$$

where $\mu=m / M$.

Developing the same transformations as for the nonlocalized mode with respect to the singular points of (3.11) and its exponents, one obtains that the boundary solutions have the form of the power series (3.6) or (3.7). 
Substituting these series into (3.11), one has two systems of recurrent algebraic equations to obtain the series coefficients:

$$
\begin{aligned}
& x^{0}: \frac{4}{\mu} h a_{2}+\omega^{2} a_{0}=0, \\
& x^{1}:-\frac{1}{\mu} \gamma a_{1}+\omega^{2} a_{1}+\frac{12}{\mu} h a_{3}=0, \\
& x^{2}:-\frac{4}{\mu} \gamma a_{2}+\frac{24}{\mu} h a_{4}+\omega^{2} a_{2}=0, \\
& x^{3}: \omega^{2} a_{3}+\frac{40}{\mu} h a_{5}-\frac{9}{\mu} \gamma a_{3}=0, \\
& \vdots \\
& x^{0}:-\frac{2}{\mu} X_{0}{ }^{2} h b_{0}+X_{0}{ }^{4} \omega^{2} b_{0}+\frac{4}{\mu} X_{0}{ }^{4} h b_{2}=0, \\
& x^{1}:-\frac{1}{\mu} X_{0}{ }^{4} \gamma b_{1}-\frac{6}{\mu} X_{0}{ }^{2} h b_{1}+X_{0}{ }^{4} \omega^{2} b_{1}+\frac{12}{\mu} X_{0}{ }^{4} h b_{3}=0, \\
& x^{2}:-\frac{4}{\mu} X_{0}{ }^{4} \gamma b_{2}-\frac{18}{\mu} X_{0}{ }^{2} h b_{2}+X_{0}{ }^{4} \omega^{2} b_{2}+\frac{24}{\mu} X_{0}{ }^{4} h b_{4}+-2 X_{0}{ }^{2} \omega^{2} b_{0}+\frac{2}{\mu} X_{0}{ }^{2} \gamma b_{0}=0, \\
& x^{3}:-\frac{9}{\mu} X_{0}{ }^{4} \gamma b_{3}-\frac{38}{\mu} X_{0}{ }^{2} h b_{3}+X_{0}{ }^{4} \omega^{2} b_{3}+\frac{40}{\mu} X_{0}{ }^{4} h b_{5}-2 X_{0}{ }^{2} \omega^{2} b_{1}+\frac{4}{\mu} h b_{1}+\frac{6}{\mu} X_{0}{ }^{2} \gamma b_{1}=0,
\end{aligned}
$$

Each of these systems is decomposed into two systems. One of these systems corresponds to even powers of $x$ and the other one to odd powers. The systems of linear equations have nontrivial solutions if determinants are equal to zero. The determinants are calculated up to sixth order. Thus one has four equations to determine the boundary of instability, which depend on the system parameters. The boundaries for localized mode, with the following system parameters: $\varepsilon m=0.1, M=1, \omega^{2}=1$, are shown in Figure 3.2. The regions of instability are shaded in this figure. Note that boundaries of the stability/instability regions are almost the same for values of the parameter $\varepsilon m$ which are less than 0.1 .

Besides, here examples of the unstable nonlocalized and stable localized nonlinear normal modes from the stability/instability regions (see Figure 3.2) are presented. Both vibration modes were calculated numerically if the small dissipation terms of the form $\delta \dot{x}$ and $\delta \dot{y}$ were introduced to the first and second equations (2.1), respectively. The calculations were made for the following values of the system parameters: $\varepsilon m=0.1, M=1$, $\omega=0.3, \delta=0.005$. Here $\gamma=6$ for the stable nonlocalized mode (Figure 3.3(a)) and $\gamma=$ 0.5 for the unstable nonlocalized mode (Figure 3.3(b)). Trajectories of the NNMs in the system configuration place are shown for the following time of calculation: $50 \leq t \leq 100$. 


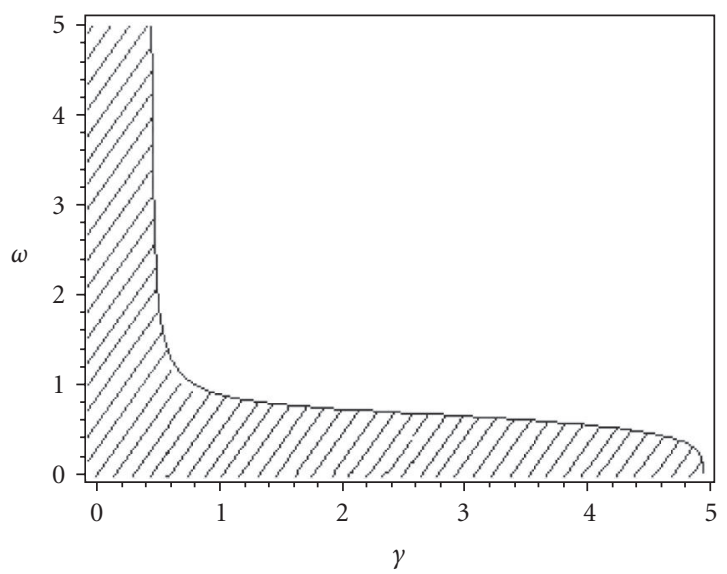

(a)

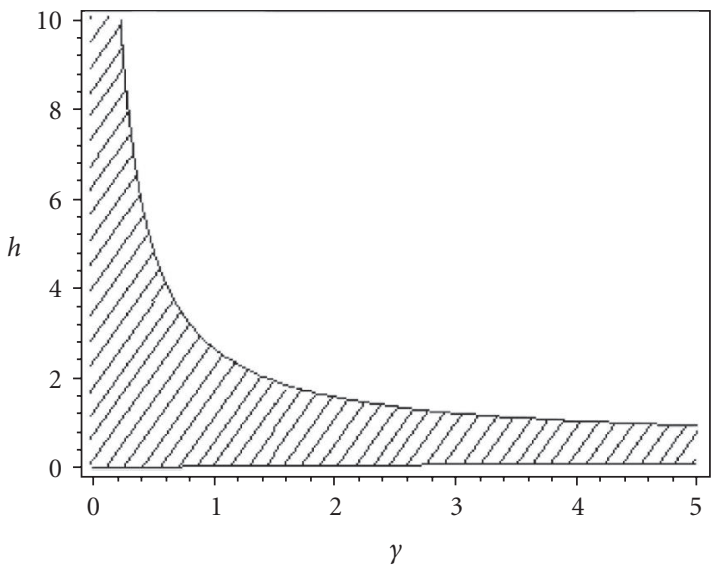

(b)

Figure 3.1. Regions of instability of the nonlocalized mode in two different planes of the system parameters, obtained by using the Ince algebraization (a) $X_{0}=1, M=1, \varepsilon m=0.1$. (b) $X_{0}=1, \omega=1$, $M=1, \varepsilon m=0.1$.

\section{Conclusions}

In this paper, the two-DOF system consisting of the linear oscillator with a relatively big mass, which is an approximation of some continuous elastic system, and the vibroimpact oscillator with a relatively small mass, which is an absorber of the main linear system vibrations, is analyzed by using the nonlinear normal mode theory. The method of nonsmooth transformation by Pilipchuk and the Ince algebraization were successfully used to obtain the frequency response and regions of stability/instability of the vibration modes. It is shown that there are large regions of the system parameters favorable for the 
12 Elastic system and a vibro-impact absorber

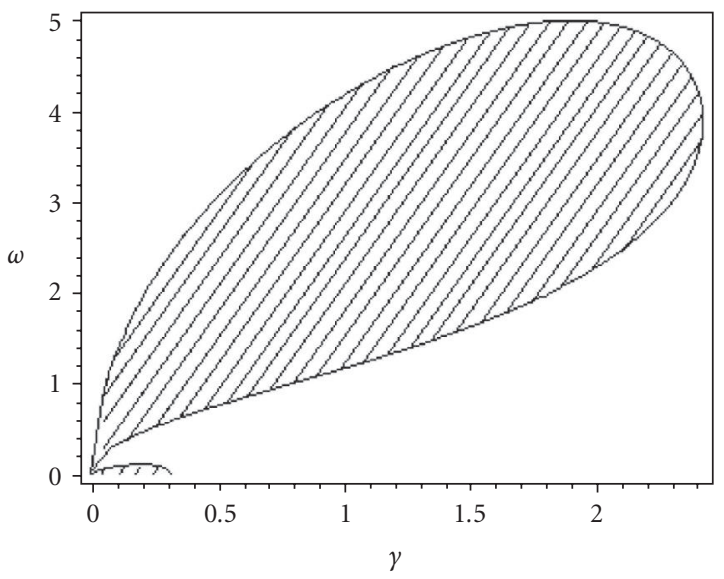

(a)

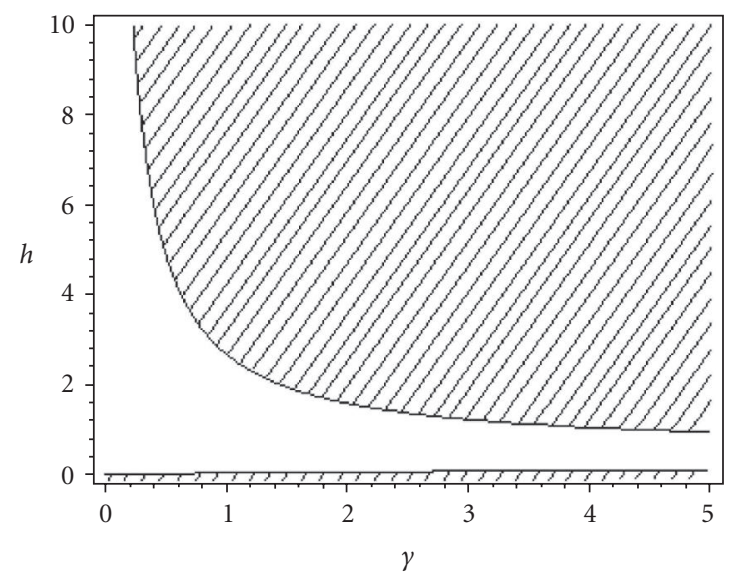

(b)

Figure 3.2. Regions of instability of the localized mode in two different planes of the system parameters, obtained by using the Ince algebraization. (a) $X_{0}=1, M=1, \varepsilon m=0.1$. (b) $X_{0}=1, \omega=1, M=1$, $\varepsilon m=0.1$.

extinguishing of elastic vibrations where the nonlocalized mode is unstable and the localized mode is stable. In a case when the localized mode, appropriate for the absorption, is realized, the main elastic system and absorber have small and significant amplitudes, respectively.

\section{Acknowledgment}

The authors are very grateful to Dr. K. V. Avramov (NTU "Kharkov Polytechnical Institute") for the useful discussions of the obtained results. 


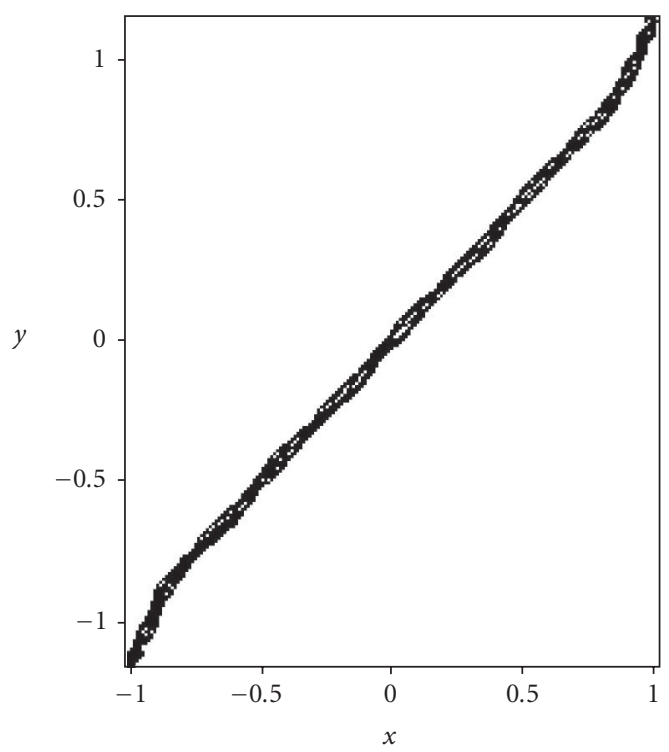

(a)

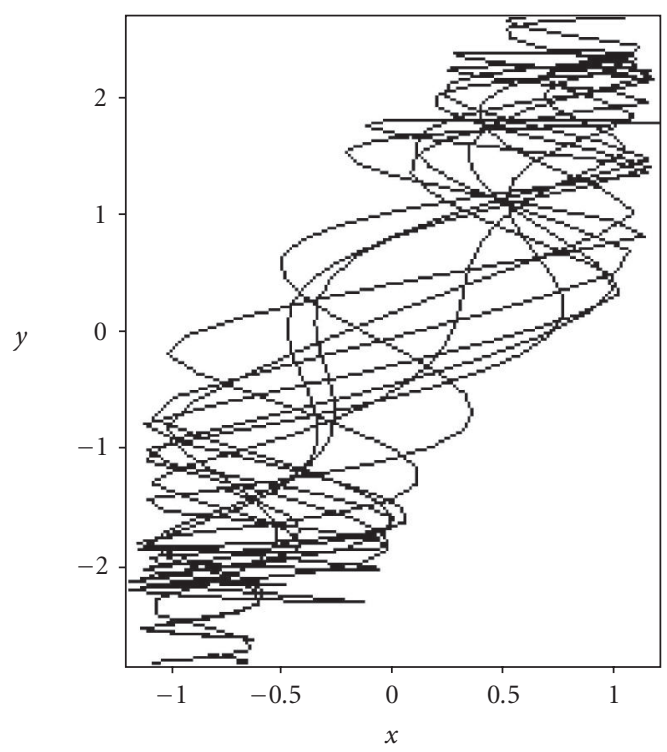

(b)

Figure 3.3. (a) Trajectory in the configuration space of the stable nonlocalized normal mode $(\varepsilon m=$ $0.1, M=1, \omega=0.3, \delta=0.005, \gamma=6$ ). (b) Trajectory in the configuration space of the unstable nonlocalized normal mode ( $\varepsilon m=0.1, M=1, \omega=0.3, \delta=0.005, \gamma=0.5)$. 


\section{References}

[1] S. Aoki and T. Watanabe, An investigation of an impact vibration absorber with hysteretic damping, Proceedings of 2nd International Conference on Motion and Vibration Control (Yokohama, 1994), 1994, pp. 468-474.

[2] K. V. Avramov and Yu. V. Mikhlin, Forced oscillations of a system, containing a snap-through truss, close to its equilibrium position, Nonlinear Dynamics. An International Journal of Nonlinear Dynamics and Chaos in Engineering Systems 35 (2004), no. 4, 361-379.

[3] _ Snap-through truss as a vibration absorber, Journal of Vibration and Control 10 (2004), no. 2, 291-308.

[4] O. Cuvalci and A. Ertas, Pendulum as vibration absorber for flexible structures: experiments and theory, ASME Journal of Vibration and Acoustics 118 (1996), no. 4, 558-566.

[5] K. V. Frolov (ed.), Vibrations in Engineering, Mashinostroenie, Moscow, 1995.

[6] A. G. Haddow and S. W. Shaw, An experimental study of torsional vibration absorbers, Proceedings of 18th Biennial Conference on Mechanical Vibration and Noise (Pennsylvania, 2001), ASME Paper DETC2001/VIB-21754, 2001, pp. 1-7.

[7] R. S. Haxton and A. D. S. Barr, The autoparametric vibration absorber, Journal of Engineering for Industry 94 (1972), 119-225.

[8] E. L. Ince, Ordinary Differential Equations, Longmans Green, London, 1926.

[9] M. P. Karyeaclis and T. K. Caughey, Stability of a semi-active impact damper. I: global behavior, American Society of Mechanical Engineers. Transactions of the ASME. Journal of Applied Mechanics 56 (1989), no. 4, 926-929.

[10] _ Stability of a semi-active impact damper. II: periodic solutions, American Society of Mechanical Engineers. Transactions of the ASME. Journal of Applied Mechanics 56 (1989), no. 4, 930-940.

[11] M. Z. Kolovski, Nonlinear Theory of Vibro-Absorber Systems, Nauka, Moscow, 1966.

[12] C.-T. Lee and S. W. Shaw, Torsional vibration reduction in internal combustion engines using centrifugal pendulums, Proceedings of ASME Design Engineering Technical Conference, Vol. 3A (Massachusetts, 1995), vol. DE-84-1, 1995, pp. 487-492.

[13] L. I. Manevich, Yu. V. Mikhlin, and V. N. Pilipchuk, The Method of Normal Oscillations for Essentially Nonlinear Systems, Nauka, Moscow, 1989.

[14] L. I. Manevitch, O. Gendelman, A. I. Musienko, A. F. Vakakis, and L. Bergman, Dynamic interaction of a semi-infinite linear chain of coupled oscillators with a strongly nonlinear end attachment, Physica D. Nonlinear Phenomena 178 (2003), no. 1-2, 1-18.

[15] D. M. McFarland, L. A. Bergman, A. F. Vakakis, L. I. Manevitch, and O. Gendelman, Energy pumping into passive nonlinear energy sinks attached to forced linear substructures: analytical and experimental results, Proceedings of 9th Conference on Nonlinear Vibrations, Stability, and Dynamics of Structures (Virginia, 2002), Virginia Polytechnic Institute and State University, 2002.

[16] Yu. V. Mikhlin, Normal vibrations of a general class of conservative oscillators, Nonlinear Dynamics. An International Journal of Nonlinear Dynamics and Chaos in Engineering Systems 11 (1996), no. 1, 1-15.

[17] Yu. V. Mikhlin and S. N. Reshetnikova, Dynamical interaction of an elastic system and essentially nonlinear absorber, Journal of Sound and Vibration 283 (2005), no. 1-2, 91-120.

[18] Yu. V. Mikhlin and A. L. Zhupiev, An application of the Ince algebraization to the stability of the non-linear normal vibration modes, International Journal of Non-Linear Mechanics 32 (1997), no. 2, 393-409.

[19] S. Natsiavas, Steady state oscillations and stability of non-linear dynamic vibration absorbers, Journal of Sound and Vibration 156 (1992), no. 2, 227-245.

[20] Vibration absorbers for a class of self-excited mechanical systems, American Society of Mechanical Engineers. Transactions of the ASME. Journal of Applied Mechanics 60 (1993), no. $2,382-387$. 
[21] V. N. Pilipchuk, Analytical study of vibrating systems with strong non-linearities by employing saw-tooth time transformations, Journal of Sound and Vibration 192 (1996), no. 1, 43-64.

[22] S. W. Shaw and S. Wiggins, Chaotic motions of a torsional vibration absorber, American Society of Mechanical Engineers. Transactions of the ASME. Journal of Applied Mechanics 55 (1988), no. 4, 952-958.

[23] A. F. Vakakis, An overview of motion localization and energy pumping in coupled nonlinear oscillators, Proceedings of 6th Conference on Dynamical Systems, Theory and Application (Lodz, 2001), 2001, pp. 111-118.

[24] __ Inducing passive nonlinear energy sinks in vibrating systems, ASME Journal of Vibration and Acoustics 123 (2001), no. 3, 324-332.

[25] A. F. Vakakis, L. I. Manevitch, Yu. V. Mikhlin, V. N. Pilipchuk, and A. A. Zevin, Normal Modes and Localization in Nonlinear Systems, Wiley Series in Nonlinear Science, John Wiley \& Sons, New York, 1996.

[26] A. Vyas and A. K. Bajaj, Dynamics of autoparametric vibration absorbers using multiple pendulums, Journal of Sound and Vibration 246 (2001), no. 1, 115-135.

Y. V. Mikhlin: National Technical University, "Kharkov Polytechnical Institute,"

Kharkov 61002, Ukraine

E-mail address: muv@kpi.kharkov.ua

S. N. Reshetnikova: National Technical University, "Kharkov Polytechnical Institute,"

Kharkov 61002, Ukraine

E-mail address: s.n.resetnokova@mail.ru 


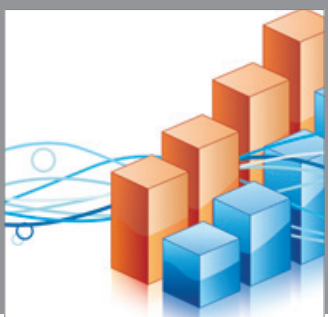

Advances in

Operations Research

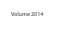

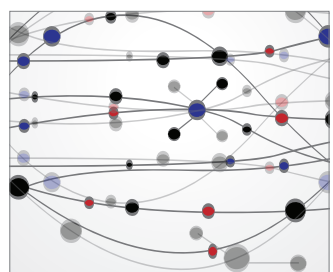

\section{The Scientific} World Journal
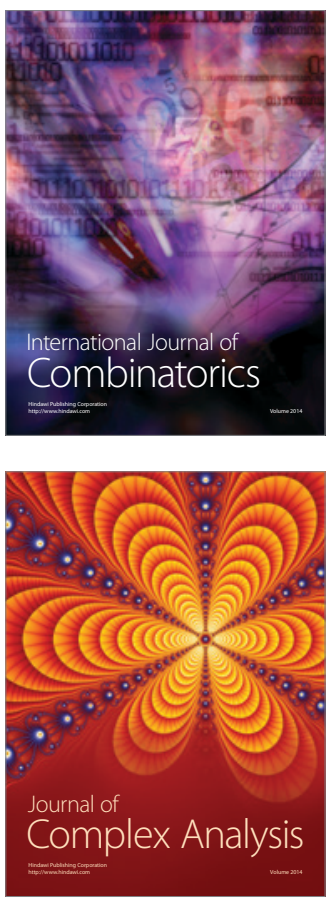

International Journal of

Mathematics and

Mathematical

Sciences
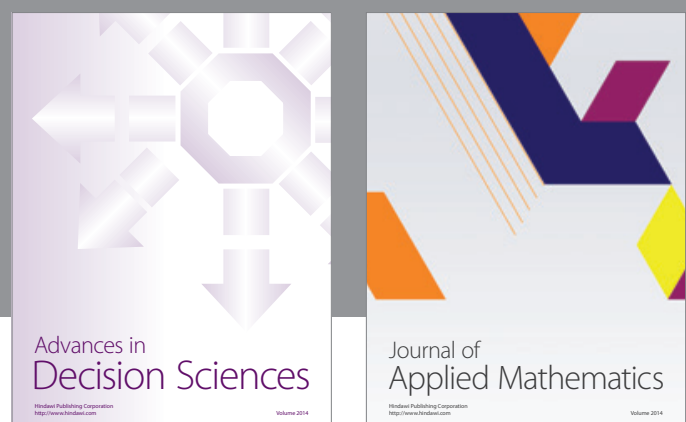

Journal of

Applied Mathematics
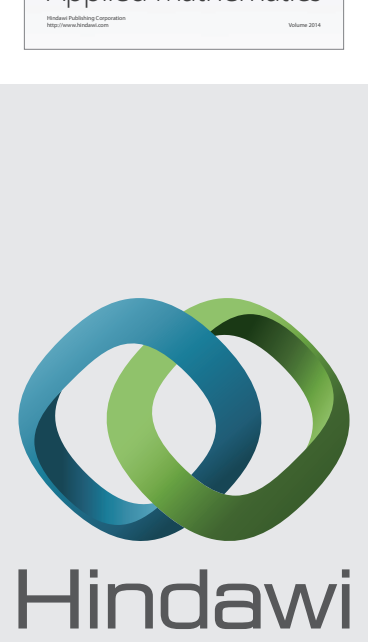

Submit your manuscripts at http://www.hindawi.com
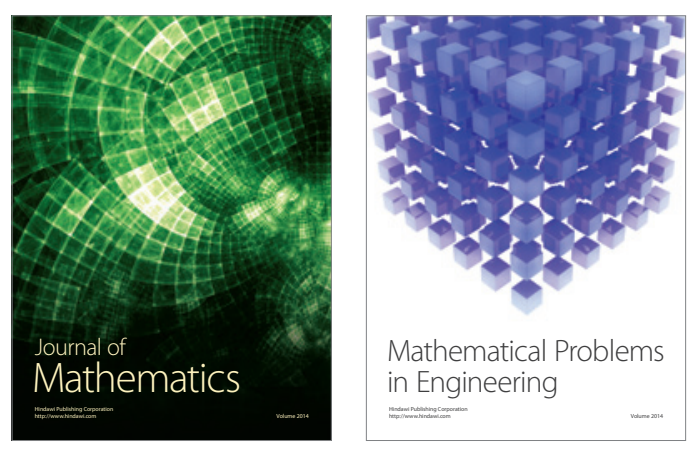

Mathematical Problems in Engineering
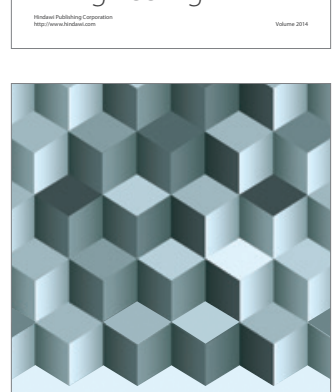

Journal of

Function Spaces
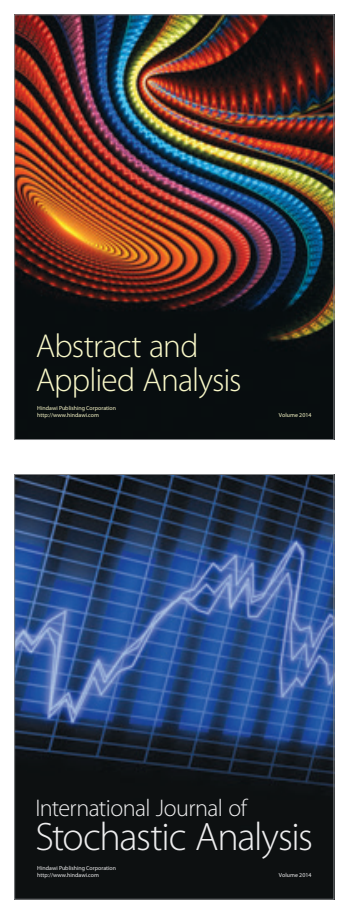

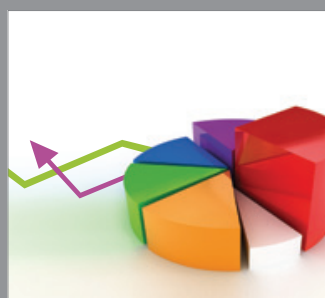

ournal of

Probability and Statistics

Promensencen
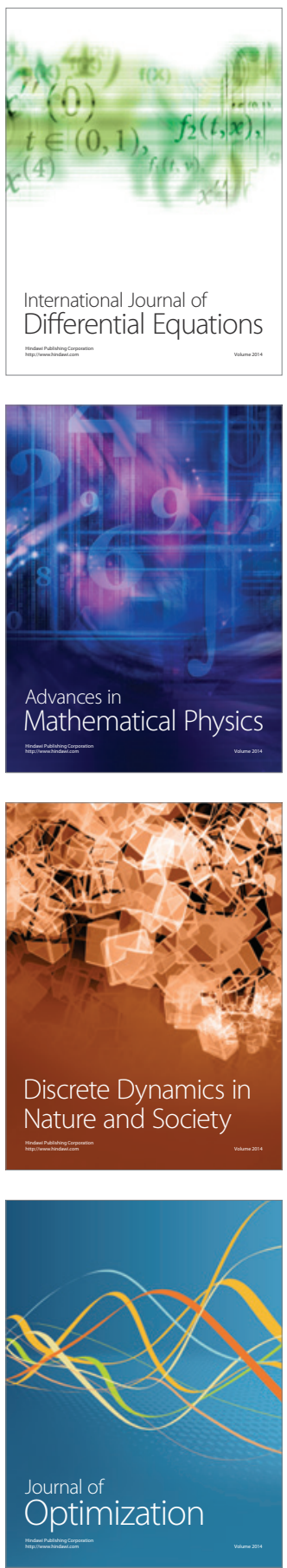